\title{
Small cell carcinoma of the bladder in transplant recipients: a report of 2 cases
}

\author{
Devendar Katkoori, MD, MS, MCh(Urol); ${ }^{*}$ Brian L. Cohen, MD, MPH; ${ }^{\dagger}$ Mark S. Soloway, MD, ; \\ Murugesan Manoharan, MD, FRCS, FRACS $(U \mathrm{rol})^{\S}$
}

\begin{abstract}
Small cell carcinoma (SCC) of the urinary bladder is a rare disease accounting for $0.5 \%$ to $0.7 \%$ of all primary bladder cancers. Transplant recipients are a special subset of patients with increased risk for various urologic malignancies, including transitional cell carcinoma of the bladder. However, to the best of our knowledge, a SCC of the urinary bladder has not been reported in transplant recipients. We report what we believe are the first 2 reported cases of transplant recipients with SCC of the bladder. Small cell carcinoma was diagnosed 5 years after transplantation in both patients and they died due to metastatic SCC. Our report emphasizes the highly aggressive nature of SCC and also the rapid progression seen in transplant recipients.
\end{abstract}

Can Urol Assoc J 2010;4(1):E4-E6

\section{Introduction}

Small cell carcinoma (SCC) occurs primarily in the lung and much less commonly in the urinary bladder, accounting for only $0.5 \%$ to $0.7 \%$ of all primary bladder cancers. Small cell carcinoma of urinary bladder is much more aggressive than transitional cell carcinoma (TCC) of the bladder, and is generally believed to have a high metastatic potential. Transplant patients have been documented to be at increased risk for several malignancies, including TCC of the bladder. However, to the best of our knowledge, a SCC of the urinary bladder has not been reported in transplant recipients. We report what we believe are the first 2 cases.

\section{Case 1}

A 73-year-old male, with a history of smoking 50 packs per year, underwent a cadaveric renal transplant in 1997 for chronic renal failure. His immunosuppressive regimen included methylprednisolone, tacrolimus, and mycophenolate mofetil. In November 2003, the patient presented with hematuria and a transurethral resection (TUR) that showed T1 TCC. He did not receive intravesical chemother- apy. In April 2004, he underwent a second TUR demonstrating high grade T2 TCC. The patient was referred to us and on slide consultation by a genitourinary pathologist; the TUR specimen was reported as consistent with a diagnosis of SCC. A radical cystectomy with neobladder was performed in May 2004. Cystectomy pathology showed small uniform cells with scant cytoplasm (Fig. 1), frequent mitotic figures and areas of necrosis. Immunohistochemistry was positive for synaptophysin (Fig. 2). The final diagnosis was SCC infiltrating into the perivesical fat without any lymphnode metastasis (T3 N0). He was subsequently counselled regarding adjuvant chemotherapy which he declined. He developed pulmonary recurrence in November 2004 and expired in February 2005.

\section{Case 2}

A 65-year-old male underwent orthotopic liver transplantation in 1999 for cirrhosis secondary to alcohol abuse. His medications included tacrolimus for immunosuppression. In June 2004, he presented with gross hematuria and underwent TUR. The patient was then referred to our institution in July 2004 and underwent a re-staging resection. Pathology demonstrated high grade T2 TCC and SCC. He was referred to a medical oncologist and received chemotherapy (carboplatin and etoposide). He subsequently developed pulmonary metastases in 5 months and expired in July 2005.

\section{Discussion}

Malignancy following transplantation is a well-recognized complication. A small proportion of these are urological malignancies, of which renal cancer is the most common. ${ }^{1}$ Most bladder cancers in transplant recipients are TCC, and the disease follows an aggressive course. ${ }^{2}$ An SCC of the bladder in the post-transplant setting has not been reported so far.

The first case of SCC of urinary bladder was reported in 1981. ${ }^{3}$ Since then, several cases have been reported. Gross hematuria with or without dysuria is the most common symp- 


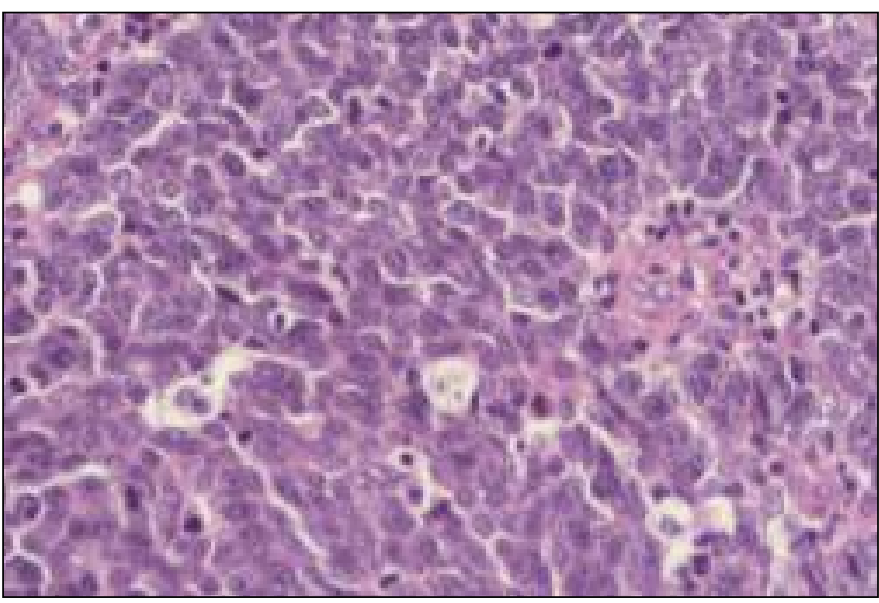

Fig. 1. Hematoxylin and eosin stain ( $\mathrm{H} \& \mathrm{E}$ ) of a radical cystectomy specimen demonstrating small cell carcinoma of the bladder.

tom. Most patients are men and most are smokers. The bladder SCC is most commonly a solid polypoidal mass. Histologically, a SCC of the bladder is indistinguishable from a SCC of the lung. Sheets or nests of small or intermediate cells with nuclear molding, scant cytoplasm, inconspicuous nucleoli and a "salt and pepper chromatin" is the hallmark appearance of SCC on light microscopy. ${ }^{4}$ Immunohistochemistry staining for neural markers, like neuron specific enolase, chromogrannin A, synaptophysin, can help in the diagnosis. Numerous theories have been proposed to explain the origin of extrapulmonary SCC. The most accepted explanation is a multipotential stem cell origin with ability to differentiate into various tissue types. The management guidelines for SCC of the bladder are still unclear. Combinations of surgical resection, chemotherapy and radiotherapy are presently used. Radical cystectomy alone has a limited role and may be used only in small volume disease. Neoadjuvant chemotherapy followed by radical cystectomy may be used where the tumour burden is limited. The high rate of systemic relapse has prompted the use of chemoradiotherapy with bladder preservation protocol. ${ }^{5}$ Stage, performance status and the use of chemotherapy are the prognostic determinants.

In a post-transplant setting, primary SCC of prostate has been reported. ${ }^{6}$ Donor derived SCC of liver and adrenal have also been previously reported. ${ }^{7-9}$ A donor derived malignancy is a very rare occurrence with modern day screening. The timing of donor derived malignancies have manifested anywhere from several days to 38 months. ${ }^{10} \mathrm{We}$ believe that in both of our patients the SCC was a primary bladder tumour, in view of the long interval between the transplant and manifestation of the bladder SCC. In the post-transplant setting and associated immunosuppression the disease usually progresses rapidly. The first patient had surgery alone and the second patient received chemotherapy following TURBT, however both developed pulmonary

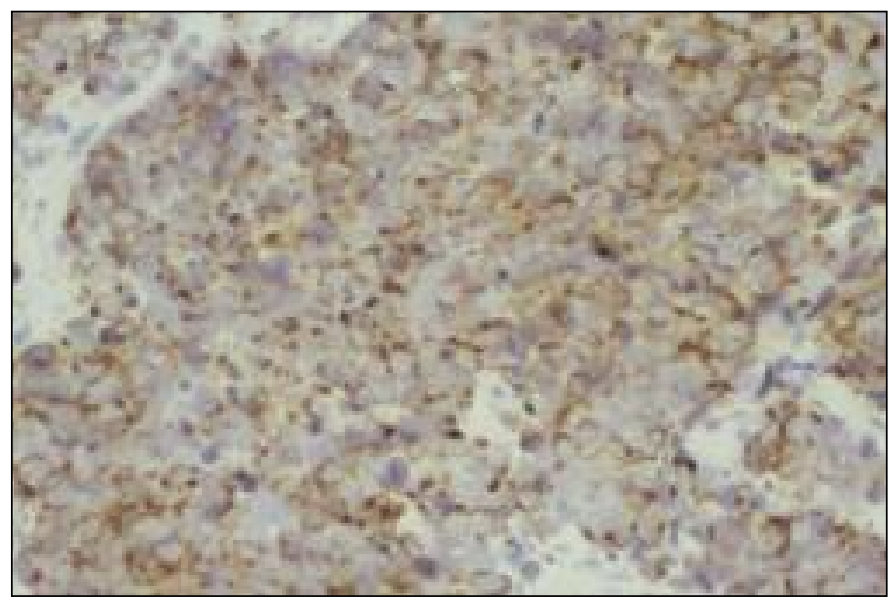

Fig. 2. Positive synaptophysin stain confirming small cell carcinoma in a radical cystectomy specimen.

metastases. The first patient in our report had a histological diagnosis of high-grade TCC on 2 occasions before being diagnosed as SCC. Immunohistochemical staining of the TUR specimens was not performed. The management of post-transplant patients with malignancy is complicated. In view of the need for a specialized care, it is of utmost importance that urologists, oncologists and transplant physicians work together to treat this complex problem in transplant recipients.

\section{Conclusion}

Our report demonstrates the aggressive nature of SCC and a rapid progression seen in transplant recipients.

${ }^{*}$ Senior research associate, Department of Urology; ${ }^{\dagger}$ Clinical Fellow in Female Urology, Voiding Dysfunction and Reconstructive Urology; §Professor and Chairman, Department of Urology; §Associate Professor, Department of Urology, Miller School of Medicine, University of Miami, Miami, FL

Acknowledgements: The authors acknowledge the support from "CURED" and Mr. Vincent A. Rodriguez.

Competing interests: None declared.

This paper has been peer-reviewed.

\section{References}

1. Besarani D, Cranston D. Urological malignancy after renal transplantation. BJU Int 2007;100:502-5.

2. Gifford RR, Wofford JE, Edwards WG Jr. Carcinoma of the bladder in renal transplant patients. A case report and collective review of cases. Clin Transplant 1998;12:65-9.

3. Cramer SF, Aikawa M, Cebelin M. Neurosecretory granules in small cell invasive carcinoma of the urinary bladder. Cancer 1981;47:724-30.

4. Wang X, MacLennan GT, Lopez-Beltran A, et al. Small cell carcinoma of the urinary bladder-histogenesis, genetics, diagnosis, biomarkers, treatment, and prognosis. Appl Immunohistochem Mol Morphol 2007;15: 8-18. 
Katkoori et al.

5. Mukesh M, Cook N, Hollingdale AE, et al. Small cell carcinoma of the urinary bladder: a 15-year retrospective review of treatment and survival in the Anglian Cancer Network. BJU Int 2009;103:747-52.

6. Rannikko A, Krogerus $L$, Nyberg $M$, et al. Rapid progression of small cell carcinoma in a renal transplant recipient. Int I Urol 2006;13:817-9.

7. Baehner $R$, Magrane $G$, Balassanian $R$, et al. Donor origin of neuroendocrine carcinoma in 2 transplant patients determined by molecular cytogenetics. Hum Pathol 2000;31:1425-9.

8. Nair BT, Bhat SH, Narayan UV, et al. Donate organs not malignancies: postoperative small cell lung carcinoma in a marginal living kidney donor. Transplant Proc 2007;39:3477-80.
9. Bodvarsson S, Burlingham W, Kusaka $S$, et al. Donor-derived small cell lung carcinoma in a kidney transplant recipient. Cancer 2001;92:2429-34.

10. Penn I. Overview of the problem of cancer in organ transplant recipients. Ann Transplant 1997;2:5-6.

Correspondence: Dr. Murugesan Manoharan, Associate Professor, Department of Urology, University of Miami Miller School of Medicine, P.0. Box 016960 (M814), Miami, FL 33101; fax: 305243-4653; mmanoharan@med.miami.edu 